\title{
Quantum stochastic Weyl operators
}

\section{Luigi Accardi}

Centro Vito Volterra, Universitá di Roma TorVergata

Via di TorVergata, 00133 Roma, Italy

volterra@volterra.mat.uniroma2.it

\section{Andreas Boukas}

Department of Mathematics, American College of Greece

Aghia Paraskevi, Athens 15342, Greece

gxk-personnel@ath.forthnet.gr 


\section{Contents}

1. Introduction

2. First order and square of white noise Weyl operators

3

3. Module form of Weyl evolutions

7

4. Matrix elements

References 


\section{Thanks}

. The second author wishes to express his gratitude to Professor Luigi Accardi for his support and guidance over the years, as well as for the hospitality of the Centro Vito Volterra of the Universita di Roma TorVergata on many occasions.

Class of Subject: Primary $81 \mathrm{~S} 25$

Abstract. The quantum stochastic differential equation satisfied by the unitary operator process $U=$ $\left\{U(t)=e^{i E(t)}: t \geq 0\right\}$ where $E(t)=\lambda t+z B_{t}^{-}+\bar{z} B_{t}^{+}+k M_{t}$, and $B_{t}^{-}, B_{t}^{+}, M_{t}$ are the square of white noise processes of [AcLuVo99], is obtained in the module form of [AcBou03b].

\section{Introduction}

Classical (i.e Itô [Ito51]) and quantum (i.e Hudson Parthasarathy [HuPa84]) stochastic calculi were unified by Accardi, Lu, and Volovich in [AcLuVo99] in the context of Hida white noise theory [Hida92], [Kuo96]. The theory was extended to include the newly discovered "square of white noise" with the use of renormalization techniques.

The problem of the unitarity of the solutions of quantum stochastic differential equations (QSDE) driven by first order white noise (related to the oscillator algebra of the Heisenberg-Weyl Lie algebra) was solved by Hudson and Parthasarathy in [HuPa84] who proved that a unitary evolution $\{U(t): t \geq 0\}$ defined in the tensor product of a "system" Hilbert space and a "noise" Boson-Fock space, satisfies a QSDE of the form

$$
d U(t)=U(t)\left[\left(i H-\frac{1}{2} L^{*} L\right) d t-L^{*} W d A(t)+L d A^{\dagger}(t)+(W-I) d \Lambda(t)\right]
$$

where $H, L$, and $W$ are bounded system space operators, with $H$ self-adjoint and $W$ unitary. Here $d A(t), d A^{\dagger}(t)$, and $d \Lambda(t)$ are the quantum stochastic differentials of the "annihilation", "creation", and "conservation" processes respectively.

The corresponding problem for "square of white noise" evolutions (related to the $\operatorname{sl}(2 ; \mathbb{R})$ Lie algebra) was open for several years. Preliminary work was done by Accardi, Hida, Boukas, and Kuo in [AcBou01a-g], [AcBou00a], and [AcHiKu01]. The subject was brought to a close by Accardi and Boukas in [AcBou03b] where it was shown that square of white noise unitary evolutions satisfy QSDE of the type

$$
d U_{t}=\left(\left(-\frac{1}{2}\left(D_{-} \mid D_{-}\right)+i H\right) d t+d \mathcal{A}_{t}\left(D_{-}\right)+d \mathcal{A}_{t}^{\dagger}\left(-l(W) D_{-}\right)+d \mathcal{L}_{t}(W-I)\right) U_{t}
$$

formulated on the module $\mathcal{B}\left(\mathcal{H}_{S}\right) \otimes \Gamma(\mathcal{K})$, where $\mathcal{H}_{S}$ is a system Hilbert space, $\mathcal{K}=l_{2}(\mathbb{N})$ and $\Gamma(\mathcal{K})$ denotes the Fock space over $\mathcal{K}$ (see section 3 below and [AcBou03b] for notation and details).

Applications of quantum stochastic calculus to the control of quantum evolution and Langevin equations can be found in [AcBou03a], [AcBou02a-b], [Bou94a-b], [Bou93], [Bou96].

In this paper we take a closer look at the "Weyl" unitary operator process $U=\left\{U(t)=e^{i E(t)}: t \geq 0\right\}$ where $E(t)$ is a linear combination of time and the basic noise processes in both the first power and square of white noise cases. In the square of white noise case, we put the corresponding QSDE in the module form of $[$ AcBou03b] mentioned above.

We also find explicit formulas for the "matrix elements" of $E(t)$.

\section{First ORDER AND SQUARE OF White NOISE WEYL OPERATORS}

Let $U(\operatorname{sl}(2 ; \mathbb{R}))$ denote the universal enveloping algebra of $\operatorname{sl}(2 ; \mathbb{R})$ with generators $B^{+}, M, B^{-}$satisfying the commutation relations

$$
\left[B^{-}, B^{+}\right]=M \quad, \quad\left[M, B^{+}\right]=2 B^{+} \quad, \quad\left[M, B^{-}\right]=-2 B^{-}
$$

with involution 


$$
\left(B^{-}\right)^{*}=B^{+} \quad, \quad M^{*}=M
$$

Fixing an orthonormal basis $\left\{e_{n}, n=0,1,2, \cdots\right\}$ of $l_{2}(\mathbb{N})$, and defining the mapping

$$
\rho^{+}: U(\operatorname{sl}(2 ; \mathbb{R})) \rightarrow L\left(l_{2}(\mathbb{N})\right)\left(=\text { linear, densely defined operators on } l_{2}(\mathbb{N})\right)
$$

by

$$
\rho^{+}\left(B^{+^{n}} M^{k} B^{-l}\right) e_{m}=\theta_{n, k, l, m} e_{n+m-l}
$$

where

$$
\begin{gathered}
\theta_{n, k, l, m}=H(n+m-l) \sqrt{\frac{m-l+n+1}{m+1}} 2^{k}(m-l+1)_{n}(m+1)^{(l)}(m-l+1)^{k} \\
H(x)=\left\{\begin{array}{cc}
1 & \text { if } x \geq 0 \\
0 & \text { if } x<0
\end{array}\right. \text { is the Heaviside function } \\
0^{0}=1,\left(B^{+}\right)^{n}=\left(B^{-}\right)^{n}=N^{n}=0, \text { for } n<0
\end{gathered}
$$

and "factorial powers" are defined by

$$
\begin{aligned}
x^{(n)}= & x(x-1) \cdots(x-n+1) \\
(x)_{n}= & x(x+1) \cdots(x+n-1) \\
& (x)_{0}=x^{(0)}=1
\end{aligned}
$$

we obtain a representation of $\operatorname{sl}(2 ; \mathbb{R})$, hence of $U(s l(2 ; \mathbb{R}))$, on $l_{2}(\mathbb{N})$. We define the basic stochastic differentials in the following:

Definition 1. For $n, k, l, m \in\{0,1, \ldots\}$,

$$
\begin{gathered}
d \Lambda_{n, k, l}(t):=d \Lambda_{t}\left(\rho^{+}\left(B^{+^{n}} M^{k} B^{-l}\right)\right) \\
d A_{m}(t):=d A_{t}\left(e_{m}\right) \\
d A_{m}^{\dagger}(t):=d A_{t}^{\dagger}\left(e_{m}\right)
\end{gathered}
$$

where $\Lambda_{t}, A_{t}$, and $A_{t}^{\dagger}$ are the conservation, annihilation, and creation operator processes of [HuPa84].

$\Lambda_{t}, A_{t}$, and $A_{t}^{\dagger}$ are associated with the four dimensional oscillator algebra obtained from the three dimensional Heisenberg-Weyl Lie algebra with basis $\left\{A^{\dagger}, A, E\right\}$, commutations

and involution

$$
\left[A, A^{\dagger}\right]=E \quad, \quad\left[E, A^{\dagger}\right]=[E, A]=0
$$

$$
(A)^{*}=A^{\dagger} \quad, \quad E^{*}=E
$$

by adding (see [AcFrSk00] for details) a hermitian element $\Lambda$ satisfying 


$$
\left[\Lambda, A^{\dagger}\right]=A^{\dagger} \quad, \quad[\Lambda, A]=-A \quad, \quad[E, \Lambda]=0
$$

The usual Hudson-Partasarathy quantum stochastic differentials of [HuPa84], corresponding to the first power of the white noise functionals, are $d A_{0}(t), d A_{0}^{+}(t)$, and $d \Lambda_{0,0,0}(t)$.

Definition 2. Let $\lambda, k \in \mathbb{R}$ and $z \in \mathbb{C}$. We call "first order white noise Weyl operator" any operator of the form

$$
E(t)=\lambda t+z A_{0}(t)+\bar{z} A_{0}^{+}(t)+k \Lambda_{0,0,0}(t)
$$

Remark 1. E $(t)$ is also called a "Poisson-Weyl" operator. This terminology is justified by the fact that the process $\{E(t): t \geq 0\}$ is a classical Poisson process expressed in terms of Weyl operators.

The following proposition was proved in [AcBou01d] and is a standard result in the Hudson-Parthasarathy theory.

Proposition 1. Let $U(t)=e^{i E(t)}$ where $E(t)$ is as in Definition 2. Then $\{U(t): t \geq 0\}$ is a unitary process such that

(a) if $k \neq 0$ then

$$
d U(t)=U(t)\left[\left(i \lambda+\frac{|z|^{2}}{k^{2}} M\right) d t+\left(i z+\frac{z}{k} M\right) d A_{0}(t)+\left(i \bar{z}+\frac{\bar{z}}{k} M\right) d A_{0}^{+}(t)+(i k+M) d \Lambda_{0}(t)\right]
$$

where

$$
M=e^{i k}-1-i k \text {. }
$$

(b) if $k=0$ then

$$
d U(t)=U(t)\left[\left(i \lambda-\frac{|z|^{2}}{2}\right) d t+i z d A_{0}(t)+i \bar{z} d A_{0}^{+}(t)\right]
$$

The above two equations are of the form (1.1) with

$$
\begin{gathered}
W=e^{i k} I \\
L=\frac{\bar{z}}{k}\left(e^{i k}-1\right) I \\
H=\left(\lambda-\frac{|z|^{2}}{k}-\frac{i}{2} \frac{|z|^{2}}{k^{2}}\left[e^{i k}-e^{-i k}-1\right]\right) I
\end{gathered}
$$

and

$$
H=\lambda I, \quad L=i \bar{z} I, \quad W=-I
$$

respectively. Here, and in what follows, $I$ denotes the identity operator in the appropriate context.

Definition 3. We call "square of white noise Weyl operator" any operator of the form

$$
\begin{gathered}
E(t)=\lambda t+z B_{t}^{-}+\bar{z} B_{t}^{+}+k M_{t} \\
=(\lambda+k) t+z A_{0}(t)+\bar{z} A_{0}^{\dagger}(t)+z \Lambda_{0,0,1}(t)+\bar{z} \Lambda_{1,0,0}(t)+k \Lambda_{0,1,0}(t)
\end{gathered}
$$

where $B_{t}^{-}, B_{t}^{+}, M_{t}$ are the square of white noise processes of [AccLuVol99] expressed in terms of the basic processes $A_{0}(t), A_{0}^{\dagger}(t), \Lambda_{0,0,1}(t), \Lambda_{1,0,0}(t), \Lambda_{0,1,0}(t)$ of [AcBou03b] (see also Definition 1). 
The following proposition was also proved in [AcBou01d].

Proposition 2. Let $U=\left\{U(t)=e^{i E(t)}, t \geq 0\right\}$ where $E(t)$ is as in Definition 3. Then $U$ is a unitary process satisfying

$d U(t)=U(t)\left[\tau(\lambda, z, k) d t+\sum_{m=0}^{+\infty}\left[a_{m}(z, k) d A_{m}(t)+\bar{a}_{m}(z, k) d A_{m}^{\dagger}(t)\right]+\sum_{0<i+j+r<+\infty} l_{i, j, r}(z, k) d \Lambda_{i, j, r}(t)\right]$ where the coefficients $\tau(\lambda, z, k), a_{m}(z, k), \bar{a}_{m}(z, k)$, and $l_{i, j, r}(z, k)$ are analytic functions of $z$ and $k$.

It was shown in $[\mathrm{AcBou} 01 \mathrm{~d}]$ that $\tau(\lambda, z, k), a_{m}(z, k), \bar{a}_{m}(z, k)$, and $l_{i, j, r}(z, k)$ have the form

$$
\begin{gathered}
\tau(\lambda, z, k)=\sum_{n=1}^{+\infty} \tau_{n}(\lambda, z, k) i^{n} / n ! \\
a_{m}(z, k)=\sum_{n=1}^{+\infty} a_{m, n}(z, k) i^{n} / n ! \\
\bar{a}_{m}(z, k)=\overline{a_{m}(z, k)} \\
l_{i, j, r}(z, k)=\sum_{n=1}^{+\infty} l_{i, j, r, n}(z, k) i^{n} / n !
\end{gathered}
$$

where the coefficient processes $a_{m, n}(z, k), l_{i, j, r, n}(z, k)$, and $\tau_{n}(\lambda, z, k)$ can be computed with the use of the recursions

$$
a_{m, n}(z, k)=\bar{z} \theta_{0,0,1, m+1} a_{m+1, n-1}(z, k)+k \theta_{0,1,0, m} a_{m, n-1}(z, k)+z \theta_{1,0,0, m-1} a_{m-1, n-1}(z, k)
$$

with

$$
\begin{gathered}
a_{0,1}(z, k)=z, \\
\tau_{n}(\lambda, z, k)=a_{0, n-1}(z, k) \bar{z}
\end{gathered}
$$

with

$$
\tau_{1}(\lambda, z, k)=\lambda
$$

and

$$
\begin{gathered}
l_{i, j, r, n}=\bar{z} \sum \hat{c}_{\beta, \gamma, 1,0}^{r, \rho, j-\omega, \omega, 0} l_{i+\gamma-r-1, \beta, \gamma, n-1}+k \sum \hat{c}_{\beta, \gamma, 0,1}^{r, \rho, j-\omega-\epsilon, \omega, \epsilon} l_{i+\gamma-r, \beta, \gamma, n-1} \\
+z \sum \hat{c}_{\beta, \gamma, 0,0}^{r-1, \rho, j-\omega, \omega, 0} l_{i+\gamma-r+1, \beta, \gamma, n-1}
\end{gathered}
$$

with

$$
l_{1,0,0,1}=\bar{z}, \quad l_{0,1,0,1}=k, \quad l_{0,0,1,1}=z
$$

Here, as in [AcBou01d], 


$$
\begin{gathered}
\hat{c}_{\beta, \gamma, a, b}^{\lambda, \rho, \sigma, \omega, \epsilon}=\left\{\begin{array}{ll}
c_{\beta, \gamma, a, b}^{\lambda, \rho, \sigma, \omega, \epsilon} & \text { if } 0 \leq \lambda \leq \gamma, 0 \leq \rho \leq \gamma-\lambda, \\
0 & 0 \leq \sigma \leq \gamma-\lambda-\rho, 0 \leq \omega \leq \beta, 0 \leq \epsilon \leq b
\end{array}\right\} \\
\text { otherwise }
\end{gathered}
$$

$S_{\gamma-\lambda-\rho, \sigma}$ are the "Stirling numbers of the first kind", and

$$
\sum=\sum_{\lambda=0}^{\gamma} \sum_{\rho=0}^{\gamma-\lambda} \sum_{\sigma=0}^{\gamma-\lambda-\rho} \sum_{\omega=0}^{\beta} \sum_{\epsilon=0}^{b}
$$

\section{Module Form of Weyl eVolutions}

Quantum stochastic differential equations driven by the square of white noise were elegantly described in [AccBou03b] as Hudson-Partasarathy type equations on the module $\mathcal{B}\left(\mathcal{H}_{S}\right) \otimes \Gamma(\mathcal{K})$, where $\mathcal{H}_{S}$ is a system Hilbert space, $\mathcal{K}=l_{2}(\mathbb{N})$ and $\Gamma(\mathcal{K})$ denotes the Fock space over $\mathcal{K}$.

Let

$$
E(t)=(\lambda+k) t+z A_{0}(t)+\bar{z} A_{0}^{\dagger}(t)+z \Lambda_{0,0,1}(t)+\bar{z} \Lambda_{1,0,0}(t)+k \Lambda_{0,1,0}(t)
$$

as in Definition 3.

Letting

$$
T=z \otimes e_{0}
$$

and

$$
N=z \rho^{+}\left(B^{+^{0}} M^{0} B^{-1}\right)+\bar{z} \rho^{+}\left(B^{+^{1}} M^{0} B^{-0}\right)+k \rho^{+}\left(B^{+}{ }^{0} M^{1} B^{-0}\right)
$$

we can write

$$
E(t)=(\lambda+k) t+\mathcal{A}_{t}(T)+\mathcal{A}_{t}^{\dagger}(T)+\mathcal{L}_{t}(N)
$$

where the module differentials $d \mathcal{A}_{t}(\cdot), d \mathcal{A}_{t}^{\dagger}(\cdot)$, and $d \mathcal{L}_{t}(\cdot)$ were defined in [AcBou03b] and can be multiplied with the use of the Itô table of [AcBou03b], namely

$$
\begin{gathered}
d \mathcal{A}_{t}\left(D_{-}\right) d \mathcal{A}_{t}^{\dagger}\left(D_{+}\right)=\left(D_{-} \mid D_{+}\right) d t \\
d \mathcal{L}_{t}\left(D_{1}\right) d \mathcal{L}_{t}\left(E_{1}\right)=d \mathcal{L}_{t}\left(D_{1} \circ E_{1}\right) \\
d \mathcal{L}_{t}\left(D_{1}\right) d \mathcal{A}_{t}^{\dagger}\left(D_{+}\right)=d \mathcal{A}_{t}^{\dagger}\left(l\left(D_{1}\right) D_{+}\right) \\
d \mathcal{A}_{t}\left(D_{-}\right) d \mathcal{L}_{t}\left(E_{1}\right)=d \mathcal{A}_{t}\left(r\left(E_{1}\right) D_{-}\right)
\end{gathered}
$$

where

$$
\begin{gathered}
D_{+}=\sum_{n} D_{+, n} \otimes e_{n} \\
D_{-}=\sum_{m} D_{-, m} \otimes e_{m} \\
D_{1}=\sum_{\alpha, \beta, \gamma} D_{1, \alpha, \beta, \gamma} \otimes \rho^{+}\left(B^{+\alpha} M^{\beta} B^{-\gamma}\right) \\
E_{1}=\sum_{a, b, c} E_{1, a, b, c} \otimes \rho^{+}\left(B^{+^{a}} M^{b} B^{-c}\right)
\end{gathered}
$$


with $n, m, \alpha, \beta, \gamma, a, b, c \in\{0,1,2, \ldots\}, D_{+, n}, D_{-, m}, D_{1, \alpha, \beta, \gamma}, E_{1, a, b, c} \in \mathcal{B}\left(\mathcal{H}_{S}\right), r(\cdot)$ and $l(\cdot)$ the right and left module actions respectively, defined by

$$
\begin{aligned}
& l\left(D_{1}\right) D_{+}=\sum_{n, \alpha, \beta, \gamma} D_{1, \alpha, \beta, \gamma} \theta_{\alpha, \beta, \gamma, n-\alpha+\gamma} D_{+, n-\alpha+\gamma} \otimes e_{n} \\
& r\left(E_{1}\right) D_{-}=\sum_{n, \alpha, \beta, \gamma} E_{1, \alpha, \beta, \gamma}^{*} \theta_{\gamma, \beta, \alpha, n+\alpha-\gamma} D_{-, n+\alpha-\gamma} \otimes e_{n}
\end{aligned}
$$

$(\cdot \mid \cdot)$ the module inner product, defined linearly on elementary tensors by

$$
(a \otimes \xi \mid b \otimes \eta)=a^{*} b\langle\xi, \eta\rangle
$$

and $D_{1} \circ E_{1}$ defined in [AcBou03b] by

$$
D_{1} \circ E_{1}=\sum_{\alpha, \beta, \gamma} \sum_{a, b, c} \sum c_{\beta, \gamma, a, b}^{\lambda, \rho, \sigma, \omega, \epsilon} D_{1, \alpha, \beta, \gamma} E_{1, a, b, c} \otimes \rho^{+}\left(B^{+^{a+\alpha-\gamma+\lambda}} M^{\omega+\sigma+\epsilon} B^{-\lambda+c}\right)
$$

All other products of stochastic differentials (including $d t$ ) are equal to zero.

A simple form of the equation satisfied by the operator process $U=\left\{U(t)=e^{i E(t)}, t \geq 0\right\}$ of Proposition 2 can be derived as follows.

Proposition 3. Let $U=\left\{U(t)=e^{i E(t)}, t \geq 0\right\}$ where $E(t)$ is as in (3.1). Then

$$
\begin{gathered}
d U(t)=U(t)\left[(i(\lambda+k)+(T \mid f(l(N)) T)) d t+d \mathcal{A}_{t}((h(r(N))-i) T)\right. \\
\left.+d \mathcal{A}_{t}^{\dagger}((g(l(N))+i) T)+d \mathcal{L}_{t}\left(e^{\circ i N}-1\right)\right]
\end{gathered}
$$

where the analytic functions $f, g, h$ are defined by

$$
\begin{gathered}
e^{i x}=1+i x+x^{2} f(x) \\
e^{i x}=1+i x+x g(x) \\
e^{-i x}=1-i x+x h(x)
\end{gathered}
$$

and

$$
e^{\circ i N}=\sum_{n=0}^{+\infty} \frac{i^{n}}{n !} N^{\circ n}
$$

where

$$
N^{\circ n}=N \circ N \circ \cdots \circ N(n \text {-times })
$$

Remark 2. The QSDE satisfied by $U=\left\{U(t)=e^{i E(t)}, t \geq 0\right\}$ is of the form (1.2), with

$$
\begin{aligned}
& W=e^{\circ i N} \\
& H=\lambda+k
\end{aligned}
$$

and

$$
D_{-}=(h(r(N))-i) T
$$


Proof. Computing the differential of $U(t)$ we find

$$
\begin{gathered}
d U(t)=d\left(e^{i E(t)}\right)=e^{i E(t+d t)}-e^{i E(t)}=e^{i(E(d t)+E(t))}-e^{i E(t)} \\
\left.=e^{i E(d t)} e^{i E(t)}-e^{i E(t)} \text { (by the commutativity of } E(d t) \text { and } E(t)\right) \\
=e^{i E(t)}\left[e^{i d E(t)}-I\right]=U(t) \sum_{n=1}^{\infty} \frac{(i d E(t))^{n}}{n !}
\end{gathered}
$$

By the module form of the square of white noise Itô table

$$
\begin{gathered}
d E(t)^{2}=\left((\lambda+k) d t+d \mathcal{A}_{t}(T)+d \mathcal{A}_{t}^{\dagger}(T)+d \mathcal{L}_{t}(N)\right) \\
\cdot\left((\lambda+k) d t+d \mathcal{A}_{t}(T)+d \mathcal{A}_{t}^{\dagger}(T)+d \mathcal{L}_{t}(N)\right) \\
=(T \mid T) d t+d \mathcal{A}_{t}(r(N) T)+d \mathcal{A}_{t}^{\dagger}(l(N) T)+d \mathcal{L}_{t}(N \circ N) \\
d E(t)^{3}=\left((\lambda+k) d t+d \mathcal{A}_{t}(T)+d \mathcal{A}_{t}^{\dagger}(T)+d \mathcal{L}_{t}(N)\right) \\
\cdot\left((T \mid T) d t+d \mathcal{A}_{t}(r(N) T)+d \mathcal{A}_{t}^{\dagger}(l(N) T)+d \mathcal{L}_{t}(N \circ N)\right) \\
=(T \mid l(N) T) d t+d \mathcal{A}_{t}\left(r(N)^{2} T\right)+d \mathcal{A}_{t}^{\dagger}\left(l(N)^{2} T\right)+d \mathcal{L}_{t}(N \circ N \circ N)
\end{gathered}
$$

and, in general, for $n \geq 2$

$$
d E(t)^{n}=\left(T \mid l(N)^{n-2} T\right) d t+d \mathcal{A}_{t}\left(r(N)^{n-1} T\right)+d \mathcal{A}_{t}^{\dagger}\left(l(N)^{n-1} T\right)+d \mathcal{L}_{t}\left(N^{\circ n}\right)
$$

Thus

$$
\begin{gathered}
d U(t)=U(t) \sum_{n=1}^{\infty} \frac{(i d E(t))^{n}}{n !}=U(t)\left[i d E(t)+\sum_{n=2}^{\infty} \frac{(i d E(t))^{n}}{n !}\right] \\
=U(t)\left[i d E(t)+\left(T \mid \sum_{n=2}^{\infty} \frac{i^{n}}{n !} l(N)^{n-2} T\right) d t+d \mathcal{A}_{t}\left(\sum_{n=2}^{\infty} \frac{(-1)^{n} i^{n}}{n !} r(N)^{n-1} T\right)\right. \\
\left.+d \mathcal{A}_{t}^{\dagger}\left(\sum_{n=2}^{\infty} \frac{i^{n}}{n !} l(N)^{n-1} T\right)+d \mathcal{L}_{t}\left(\sum_{n=1}^{\infty} \frac{i^{n}}{n !} N^{\circ n}\right)\right] \\
=U(t)\left[(i(\lambda+k)+(T \mid f(l(N)) T)) d t+d \mathcal{A}_{t}((h(r(N))-i) T)+d \mathcal{A}_{t}^{\dagger}((g(l(N))+i) T)+d \mathcal{L}_{t}\left(e^{\circ i N}-1\right)\right]
\end{gathered}
$$

\section{Matrix Elements}

Proposition 4. Let $E(t)$ be as in Definition 2 and let $\psi(f), \psi(g)$ be two "exponential vectors" in the Boson Fock space $\Gamma\left(L^{2}([0,+\infty), \mathbb{C})\right)$, in the sense of $[H u P a 84]$. Then, for each $t \geq 0$ and $f$ and $g$ in $\left.L^{2}([0,+\infty), \mathbb{C})\right)$

$$
<\psi(f), E(t) \psi(g)>=\left[(\lambda+k) t+z \int_{0}^{t} g(s) d s+\bar{z} \int_{0}^{t} \bar{f}(s) d s+k \int_{0}^{t} \bar{f}(s) g(s) d s\right] e^{\int_{0}^{+\infty} \bar{f}(s) g(s) d s}
$$

Proof. The proof follows directly from the formulas for the matrix elements of the basic Hudson-Parthasarathy noise processes, provided in [HuPa84].

Proposition 5. Let $E(t)$ be as in Definition 3 and let $\psi(f), \psi(g)$ be two "exponential vectors" in the Boson Fock space $\Gamma\left(L^{2}\left([0,+\infty), l_{2}(\mathbb{N})\right)\right)$. Then, for each $t \geq 0$ and functions $f, g$ in $L^{2}\left([0,+\infty), l_{2}(\mathbb{N})\right)$ with $f(s)=\left\{f_{n}(s)\right\}_{n=0}^{+\infty}, g(s)=\left\{g_{n}(s)\right\}_{n=0}^{+\infty}$ and

$$
<f, g>=\sum_{n=0}^{+\infty}\left[\int_{0}^{+\infty} \bar{f}_{n}(s) g_{n}(s) d s\right]
$$




$$
\begin{gathered}
<\psi(f), E(t) \psi(g)>=\left[(\lambda+k) t+\bar{z} \int_{0}^{t} g_{0}(s) d s+z \int_{0}^{t} \bar{f}_{0}(s) d s\right. \\
+\bar{z} \sum_{n=0}^{+\infty} \sqrt{(n+2)(n+1)} \int_{0}^{t} \bar{f}_{n}(s) g_{n+1}(s) d s+z \sum_{n=0}^{+\infty} \sqrt{n(n+1)} \int_{0}^{t} \bar{f}_{n}(s) g_{n-1}(s) d s \\
\left.+k \sum_{n=0}^{+\infty}(2 n+2) \int_{0}^{t} \bar{f}_{n}(s) g_{n}(s) d s\right] e^{<f, g>}
\end{gathered}
$$

Proof. By (2.5), Definition 1, and (2.1), with $e_{n}=(0,0, \ldots, 0,1,0, \ldots)$ where 1 is in the $n+1$-st position,

$$
<\psi(f), A_{t}\left(e_{0}\right) \psi(g)>=<\psi(f),<\chi_{[0, t]} e_{0} g>\psi(g)>=\int_{0}^{t} g_{0}(s) d s<\psi(f), \psi(g)>
$$

and by the duality of $A$ and $A^{\dagger}$

$$
<\psi(f), A_{t}^{\dagger}\left(e_{0}\right) \psi(g)>=\int_{0}^{t} \bar{f}_{0}(s) d s<\psi(f), \psi(g)>
$$

Moreover

$$
\begin{gathered}
<\psi(f), \Lambda_{0,0,1}(t) \psi(g)>=<\psi(f), \Lambda\left(\chi_{[0, t]} \rho^{+}\left(B^{-}\right)\right) \psi(g)> \\
=<f, \chi_{[0, t]} \rho^{+}\left(B^{-}\right) g><\psi(f), \psi(g)>=\int_{0}^{t}<f(s), \rho^{+}\left(B^{-}\right) g(s)>d s<\psi(f), \psi(g)> \\
=\sum_{n=0}^{+\infty}\left[\int_{0}^{+\infty} \bar{f}_{n}(s)\left(\rho^{+}\left(B^{-}\right) g\right)_{n}(s) d s\right]<\psi(f), \psi(g)>
\end{gathered}
$$

and since

$$
\begin{gathered}
g(s)=\sum_{n=0}^{+\infty} g_{n}(s) e_{n} \Rightarrow \rho^{+}\left(B^{-}\right) g(s)=\sum_{n=0}^{+\infty} g_{n}(s) \sqrt{n(n+1)} e_{n-1} \\
\Rightarrow\left(\rho^{+}\left(B^{-}\right) g\right)_{n}(s)=g_{n+1}(s) \sqrt{(n+1)(n+2)}
\end{gathered}
$$

we obtain

$$
<\psi(f), \Lambda_{0,0,1}(t) \psi(g)>=\sum_{n=0}^{+\infty} \sqrt{(n+1)(n+2)}\left[\int_{0}^{t} \bar{f}_{n}(s) g_{n+1}(s) d s\right]<\psi(f), \psi(g)>
$$

Similarly

$$
<\psi(f), \Lambda_{1,0,0}(t) \psi(g)>=\sum_{n=0}^{+\infty} \sqrt{n(n+1)}\left[\int_{0}^{t} \bar{f}_{n}(s) g_{n-1}(s) d s\right]<\psi(f), \psi(g)>
$$

and

$$
<\psi(f), \Lambda_{0,1,0}(t) \psi(g)>=\sum_{n=0}^{+\infty}(2 n+2)\left[\int_{0}^{t} \bar{f}_{n}(s) g_{n}(s) d s\right]<\psi(f), \psi(g)>
$$

\section{REFERENCES}

[1] Accardi L., Boukas A.: Unitarity conditions for stochastic differential equations driven by nonlinear quantum noise, Random Operators and Stochastic Equations, 10 (1) (2002) 1-12, Preprint Volterra N. 463 (2001)

[2] Accardi L., Boukas A.: Stochastic evolutions driven by non-linear quantum noise, Probability and Mathematical Statistics, 22 (1) (2002) Preprint Volterra N. 465 (2001)

[3] Accardi L., Boukas A.: Stochastic evolutions driven by non-linear quantum noise II, Russian Journal of Mathematical Physics 8 (4) (2001) Preprint Volterra N. 467 (2001) 
[4] Accardi L., Boukas A.: Square of white noise unitary evolutions on Boson Fock space, International conference on stochastic analysis in honor of Paul Kree, Hammamet, Tunisie, October 22-27, 2001.

[5] Accardi L., Boukas A.: The semi-martingale property of the square of white noise integrators, in: proceedings of the Conference: Stochastic differential equations, Levico, january 2000, G. Da Prato, L.Tubaro (eds.), Pittman (2001) 1-19, Preprint Volterra N. 467 (2001)

[6] Accardi L., Boukas A.:Control of elementary quantum flows, Proceedings of the 5th IFAC symposium on nonlinear control systems, July 4-6, 2001, St. Petersburg, Russia".

[7] Accardi L., Boukas A., Kuo H.-H.: On the unitarity of stochastic evolutions driven by the square of white noise, Infinite Dimensional Analysis, Quantum Probability, and Related Topics 4 (4) (2001) 1-10

[8] Accardi L., Boukas A.: From classical to quantum quadratic cost control, in: Proceedings of the "International Winter School on Quantum Information and Complexity", Meijo University, Japan, Jan. 6-10, 2003.

[9] Accardi L., Boukas A.: The unitarity conditions for the square of white noise, to appear in Infinite Dimensional Analysis, Quantum Probability, and Related Topics (2003).

[10] Accardi L., Boukas A.: Unitarity conditions for the renormalized square of white noise, in: Trends in Contemporary Infinite Dimensional Analysis and Quantum Probability, Natural and Mathematical Sciences Series 3, Italian School of East Asian Studies, Kyoto, Japan (2000) 7-36, Preprint Volterra N. 405 (2000)

[11] Accardi L., Boukas A.: Quadratic control of quantum processes, Russian Journal of Mathematical Physics (2002).

[12] Accardi L., Boukas A.: Control of quantum Langevin equations, Open Systems and Information Dynamics 9 (2002) 1-15

[13] Accardi L., Franz U., Skeide M.: Renormalized squares of white noise and other non-Gaussian noises as Levy processes on real Lie algebras, Comm. Math. Phys. 228 (2002) 123-150 Preprint Volterra, N. 423 (2000)

[14] Accardi L., Hida T., Kuo H.H.: The Itô table of the square of white noise, Infinite dimensional analysis, quantum probability and related topics, 4 (2) (2001) 267-275 Preprint Volterra, N. 459 (2001)

[15] Accardi L., Lu Y.G., Volovich I.V.: White noise approach to classical and quantum stochastic calculi, Lecture Notes of the Volterra International School of the same title, Trento, Italy, 1999, Volterra Preprint N. 375 July (1999)

[16] Accardi L., Lu Y.G., Volovich I.: Quantum Theory and its Stochastic Limit, Springer Verlag (2002)

[17] Boukas A.:Linear Quantum Stochastic Control, Quantum Probability and related topics, 105-111, QP -PQ IX, World Scientific Publishing, River Edge NJ,1994.

[18] Boukas A.:Application of Operator Stochastic Calculus to an Optimal Control problem, Mat. Zametki 53 (5) (1993) 48-56 Russian edition. Translation in Math. Notes 53 (5-6) (1993) 489-494, MR 96a 81070.

[19] Boukas A.: Operator valued stochastic control in Fock space with applications to noise filtering and orbit tracking, Journal of Probability and Mathematical Statistics, 16 (1) 1994

[20] Boukas A.: Stochastic Control of operator-valued processes in Boson Fock space, Russian Journal of Mathematical Physics 4 (2) (1996) 139-150, MR 97j 81178

[21] Hida T.: Selected papers, World Scientific (2001)

[22] Hudson R.L., Parthasarathy K.R.: Quantum Ito's formula and stochastic evolutions, Commun. Math. Phys. 93 (1984) 301-323

[23] K. Ito: On stochastic differential equations, Memoirs Amer. Math. Soc. 4 (1951)

[24] Kuo H.-H.: White Noise Distribution Theory, CRC Press (1996)

[25] S. Wolfram: The Mathematica Book, 4th ed., Wolfram Media/Cambridge University Press (1999) 